\title{
STAT3 single-nucleotide polymorphisms and STAT3 mutations associated with hyper-IgE syndrome are not responsible for increased serum IgE serum levels in asthma families
}

\author{
Matthias Wjst*,1,2, Peter Lichtner ${ }^{3}$, Thomas Meitinger ${ }^{3,4}$ and Bodo Grimbacher ${ }^{5}$ \\ ${ }^{1}$ Institute of Inhalation Biology/Molecular Pneumology, Helmholtz Zentrum München, Munich-Neuherberg, Germany; \\ ${ }^{2}$ Institute of Genetic Medicine, European Academy (EURAC), Drususallee, Bolzano, Italia; ${ }^{3}$ Institute of Human \\ Genetics, Helmholtz Zentrum München, Munich-Neuherberg, Germany; ${ }^{4}$ Institute of Human Genetics, Technical \\ University Munich, München, Germany; ${ }^{5}$ Department of Immunology, Royal Free Hospital University College London, \\ London, Great Britain
}

Mutations in STAT3 (signal transducer and activator of transcription 3) have recently been found to cause the hyper-IgE syndrome (HIES) - a rare immunodeficiency syndrome including complex somatic features. We now tested whether STAT3 mutations or single-nucleotide polymorphisms (SNPs) within STAT3 may be responsible for increased IgE levels in asthmatic children. We genotyped DNA samples from 918 individuals of 217 core families by MALDI-TOF mass spectrometry. SNPs were selected from previous reports, by functional relevance and haplotype-tagging capacity. In 24 assays, including the recently described HIES mutations, no variant was detected. In another 27 SNP assays, there was no association of any STAT3 variant with asthma, allergic rhinitis or eczema. In addition, neither total and specific IgE and eosinophil count nor any lung function parameter showed any significant association. When combining high eosinophil counts and high total IgE levels to an HIES-like trait, four SNPs in the $5^{\prime}$-UTR of STAT3 were slightly overtransmitted. A minor fraction of asthmatic children may possibly have an alternate STAT3 promoter architecture influencing joined $\mathrm{IgE}$ and eosinophil upregulation. While an overall effect of STAT3 mutations on serum IgE is unlikely in asthma children.

European Journal of Human Genetics (2009) 17, 352-356; doi:10.1038/ejhg.2008.169; published online 8 October 2008

Keywords: asthma; allergy; IgE; STAT3; SNP; mutation

\section{Introduction}

The STAT (signal transducer and activator of transcription) family consists of seven members that communicate between the nucleus and the cytoplasm. ${ }^{1}$ Activated by cytokines at sites of infection and after signal transmission

*Correspondence: Dr M Wjst, Institute of Inhalation Biology, Helmholtz Zentrum München, Ingolstädter Landstrasse 1, Neuherberg, Munich D-85764, Germany.

Tel: + 49893187 3206; Fax: + 49893187 3380; E-mail: m@wjst.de Received 25 April 2008; revised 18 August 2008; accepted 27 August 2008; published online 8 October 2008 through the cell surface, STATs are activated by tyrosine phosphorylation, which serves as a molecular switch altering STAT confirmation. After trafficking to the nucleus, they bind to nuclear DNA and alter RNA transcription. ${ }^{1}$ The STATs usually have an amino terminus where they dimerize, a coiled-coil domain that interacts with other proteins like nuclear transporters, a central DNA-binding domain, a SRC homology-2 domain (SH2) that may be phosphorylated and ultimately a carboxy terminus that facilitates transcription.

Of the known STAT genes (STAT1, -2, -3, -4, -5A, -5B and -6), possibly STAT $1,{ }^{2}$ but certainly STAT $6,{ }^{3}$ has been associated with allergy. STAT6-/- mice have an impaired IL-4/IL-13 
signaling resulting in defective Th2 differentiation. In contrast, STAT3-/- mice are more difficult to examine as they are embryonically lethal. ${ }^{1}$ Tissue-specific knockout shows impaired pathogen response. STAT3 functions are pleiotropic and have seemingly contradictory roles in proliferation and differentiation. ${ }^{1}$ STAT3 was first described as being IL-6 activated and accumulating in the nucleus independent of phosphorylation; other STAT3 activators are IFN, EGF and oncostatin M; there also seems to be a close relationship to the IL-10 signal by joined SOCS3 activation. ${ }^{4}$

Although STAT3 was clinically associated primarily with tumorigenesis, ${ }^{5}$ possibly also Crohn's disease, ${ }^{6}$ there is now an increasing interest in $\operatorname{IgE}$ production as two independent reports recently described that heterozygous mutations in STAT3 cause hyper-IgE syndrome (HIES). ${ }^{7,8}$ The HIES (OMIM nos. 147060 and 234700) is a rare primary immunodeficiency syndrome defined by the clinical triad of recurrent abscesses of the skin and the lungs, severe eczema and extremely elevated levels of IgE in serum. ${ }^{9}$ It seems possible that a 'dominant negative' or 'loss-of-function' genetic effect in STAT3 leads to an overshooting $\operatorname{IgE}$ response. We therefore tested the hypothesis whether any of the observed genetic alterations seen in the HIES families or any other known singlenucleotide polymorphism (SNP) reported in STAT3 may be associated with any phenotype (ie, IgE, eosinophila, asthma, allergic rhinitis or eczema) in core families with atopy/asthma.

\section{Study population, materials and methods Study population}

The German asthma sib-pair families were collected in 26 pediatric centers in Germany and Sweden for an initial genome-wide linkage scan. ${ }^{10}$ The sample is identical as in a previous report ${ }^{11}$ except that no restriction criteria were applied here. Children were on an average 11 years old and had an average $\ln (\mathrm{IgE})$ count of $5.0 \mathrm{kU} / \mathrm{l}$ with a standard deviation of $1.8 \mathrm{kU} / \mathrm{l}^{10}$

Pulmonary function tests were performed by forced flow volume tests, and bronchial challenge was performed by a defined methacholine exposure. Briefly, pulmonary function tests were performed by forced expiration in a sitting position using a nose clip. Forced flow volume tests were performed until three reproducible loops were achieved. Of these, the trial with the maximum sum of FVC and FEV1.0 was used for the analysis. Bronchial challenge with methacholine was performed with increasing doses of 0 , $0.156,0.312,0.625,1.25,2.5,5,10$ and $25 \mathrm{mg} / \mathrm{ml}$ during five consecutive breaths with $14 \mathrm{mg}$ delivered from a de Vilbiss 646 nebulizer chamber by using a breath-triggered pump ZAN 200 (Zan, Oberthulpa, Germany). Total IgE was determined with an ELISA (Pharmacia Diagnostics, Uppsala, Sweden). Allergens tested by RAST have been reported earlier. ${ }^{10-12}$ Each study participant, including the children, signed a consent form agreeing for genetic testing. All study methods were approved in 1995 by the ethics commission of 'Nordrhein-Westfalen' and again in 2001 by 'Bayerische Landesärztekammer München'.

\section{SNP selection}

STAT3 gene position in the UCSC March 2006 map was chr17:37718869-37794039 or chromosome 17q21.2., which has been occasionally described as an allergy candidate region. ${ }^{13,14}$ STAT3 exonic sequence has a $\sim 70 \%$ sequence homology to STAT1. STAT3 occupies 75170 bases organized into 24 exons (23 of these are coding) with a protein of 770 amino acids with at least 15 splice variants known so far. At the time of writing, there were 341 SNPs known; variants Tyr705Phe and Arg609Ala (both in the SH2 domain) led to a defective phosphorylation of STAT3. As large areas of conservation were found not only in exons but also within introns, additional SNP variants have been selected at these regions as well. Linkage disequilibrium is reported to be identical in CEPH European and Yorubean African individuals with both ethnic groups showing a large LD block including exons $1-16$, followed by an area of increased recombination and another LD block including the remaining exons (Supplementary Figure 1). As indicated on the hapmap website (www.hapmap.org), the common variations may be covered by $\sim 20$ haplotype-tagging variants. In addition, there a few rare variants, ${ }^{7}$ as tested by Litonjua ${ }^{15}$ and LK Hansra (personal communication ATS, San Francisco, 2007), that have been additionally included into the genotyping program. By using functional annotation data of PupaSuite (pupasuite.bioinfo.cipf.es/), the list of SNPs was further refined (Supplementary Table 1 ).

\section{DNA preparation and genotyping}

DNA was isolated from peripheral white blood cells using Qiamp (Qiagen, Germany) or Puregene isolation kits (Gentra Systems, Minneapolis, MN, USA) and distributed on masterplates with all steps recorded by a sampletracking system. Genotyping was performed using MALDI-TOF mass spectrometry of allele-specific primer extension products generated from amplified DNA sequences (MassARRAY, SEQUENOM Inc., San Diego, CA, USA). Primers were obtained from Metabion $\mathrm{GmbH}$ (Planegg-Martinsried, Germany).

\section{Analysis \\ Statistical analyses were performed using $\mathrm{R}$ software. For each SNP, the distribution of genotypes in pseudocontrols created by combining the parental alleles not transmitted to asthma children was tested by a $\chi^{2}$-test as well as the transmission to their unaffected sibs. The standardized linkage disequilibrium coefficient $D^{\prime}$ and the correlation coefficient $R^{2}$ were calculated for each pair of SNPs by using}


Table 1 Transmission disequilibrium test of STAT3 gene variants in the German Asthma Family sample (columns 1-8)

\begin{tabular}{|c|c|c|c|c|c|c|c|c|c|c|}
\hline SNP & MAF & $M A F \%$ & Allele & Transmitted:untransmitted & OR & $\chi^{2}$ & $p T D T$ & Affected:unaffected parent & $\chi^{2}$ & $P$-value \\
\hline rs12601982 & G & 0.17 & $G: A$ & $91: 101$ & 0.9 & 0.5 & 0.471 & $16: 10$ & 1.3 & 0.257 \\
\hline rs1053004 & C & 0.38 & $\mathrm{C}: \mathrm{T}$ & $178: 160$ & 1.1 & 1.0 & 0.328 & $28: 23$ & 0.4 & 0.522 \\
\hline rs3744483 & C & 0.19 & $C: T$ & 102:112 & 0.9 & 0.5 & 0.494 & $15: 11$ & 0.6 & 0.450 \\
\hline rs12103893 & C & 0.17 & $C: T$ & $88: 105$ & 0.8 & 1.5 & 0.221 & $14: 11$ & 0.3 & 0.564 \\
\hline rs2306580 & G & 0.08 & G:C & $45: 62$ & 0.7 & 2.7 & 0.100 & $14: 10$ & 0.7 & 0.414 \\
\hline rs8069645 & G & 0.28 & $\mathrm{G}: \mathrm{A}$ & $153: 140$ & 1.1 & 0.6 & 0.448 & $21: 19$ & 0.1 & 0.758 \\
\hline rs6503695 & C & 0.32 & $\mathrm{C}: \mathrm{T}$ & $160: 151$ & 1.1 & 0.3 & 0.610 & $21: 22$ & 0.0 & 0.884 \\
\hline G3363a16 & A & 0.36 & $A: C$ & 172:159 & 1.1 & 0.5 & 0.475 & $28: 25$ & 0.1 & 0.706 \\
\hline rs6503697 & $\mathrm{T}$ & 0.28 & $\mathrm{~T}: \mathrm{A}$ & $154: 140$ & 1.1 & 0.7 & 0.414 & $21: 19$ & 0.1 & 0.758 \\
\hline rs4103200 & C & 0.28 & $C: G$ & 159:146 & 1.1 & 0.6 & 0.457 & $19: 20$ & 0.0 & 0.876 \\
\hline rs957971 & G & 0.37 & $\mathrm{G}: \mathrm{C}$ & $166: 163$ & 1.0 & 0.0 & 0.869 & $26: 22$ & 0.3 & 0.599 \\
\hline rs4621023 & $\mathrm{T}$ & 0.00 & $\mathrm{~T}: \mathrm{C}$ & $1: 1$ & 1.0 & & & $0: 0$ & & \\
\hline rs16967738 & C & 0.08 & $C: T$ & $54: 61$ & 0.9 & 0.4 & 0.514 & $14: 9$ & 1.1 & 0.297 \\
\hline rs17320971 & G & 0.08 & G:A & $54: 60$ & 0.9 & 0.3 & 0.574 & $16: 10$ & 1.4 & 0.239 \\
\hline
\end{tabular}

Case-control analysis of parents with and without asthma (columns 9-11).

the 'HAPLOVIEW' software (http://www.broad.mit.edu/ mpg/haploview). Further analyses were performed using 'PLINK' software (http://pngu.mgh.harvard.edu/ purcell/ plink), whereas variance component models for association with quantitative traits were run with 'QTDT' including permutations for exact $P$-values (http://www.sph.umich. edu/csg/abecasis/QTDT).

\section{Results}

Of the 68 SNPs initially intended to genotype, 17 could not be fully analyzed due to various reasons (primer design not possible $N=1$, primer could not be included in genotyping multiplex $N=3$, low calling genotyping rate $N=6$, no genotype call $N=6$ and obvious typing error $N=1$ ). In 24 assays, we detected no variant, whereas the resulting 27 SNP assays were further analyzed. These 27 SNP assays were obtained from 918 individuals (538 cases, 380 controls). There were 485 male and 433 female individuals; 423 founders and 495 non-founders; total genotyping rate in the remaining data set being 0.932. Eight of 918 individuals were further removed for low genotyping rate whereas two further SNPs failed frequency tests. After pruning, 25 SNPs were analyzed in 217 nuclear families, 471 individuals with both parents in 208 nuclear families, 18 individuals without both parents in nine nuclear families, 418 affected offspring trios and 78 phenotypical discordant parent pairs. Except the 1861TG/F621V variant all SNPs were in Hardy-Weinberg equilibrium both in affected and unaffected individuals.
Of the previously described HIES-associated mutations, only two fathers heterozygous for $1865 \mathrm{CT} / \mathrm{T} 622 \mathrm{I}$ were detected although with low confidence scores. Father $117 \mathrm{~V}$ had no diagnosis of asthma with no further laboratory results available; father $119 \mathrm{~V}$ had also no diagnosis of asthma, serum total IgE level of $75 \mathrm{IU} / \mathrm{l}$ and $15 \%$ eosinophils in peripheral blood.

None of the analyzed SNPs were associated with asthma nor any asthma subtype (Table 1 ). There was no association with allergic rhinitis and no association with eczema. There was also no association of seropositivity to any of the tested allergens; most importantly, there was no association with total IgE serum nor eosinophilic cell count. There was also no association with parameters of baseline lung function nor bronchial hyperreactivity.

When defining an HIES-like trait by high serum eosinophil count $(>500 / \mu \mathrm{l})$ and high total IgE levels ( $>500 \mathrm{kU} / \mathrm{l}$ ), four neighboring SNPs in the $5^{\prime}$-untranslated region showed slightly significant $P$-values (rs4103200 transmitted:untransmitted ratio 32:18, rs12949918 with 29:17, rs1026916 with 31:17 and rs4796791 with 33:18). This HIES-like trait seems to occur mainly in children $(N=68)$ with only four parents affected.

In concordance with previous linkage disequilibrium data, we detected two major LD blocks across the genomic region including STAT3. Block no. 1 including seven SNPs (23 kb) and block no. 2, another 15 SNPs (55 kb, Supplementary Figure 1). Haplotypes constructed from the most informative SNPs (Supplementary Figure 2) were not 
associated with any of the quantitative or qualitative traits analyzed when adjusted for multiple testing.

Furthermore, there was no indication of epistasis within STAT3 nor with any previously typed SNP in this sample. There was also no indication of preferentially maternal or paternal transmitted alleles.

\section{Discussion}

The discovery of mutations in the SH2- and DNA-binding domains of STAT3 being responsible for the phenotype of the HIES raised the question whether genetic changes in STAT3 would be associated with elevated IgE levels or eosinophilia seen in atopic patients. To answer this question, tested the 12 mutations reported to date in HIES and additional 39 SNPs/deletions located within the STAT3 coding region or close proximity. With one exception in the SH2 domain (T622I), the HIES mutations described recently ${ }^{7}$ could not be detected in this sample. The DNAbinding and SH2 domains of STAT3, therefore, seem to be highly conserved, which was expected given the results of Minegishi 2007 et al, ${ }^{8}$ who did not find any of their STAT3 DNA-binding site mutations in 1000 unrelated healthy individuals.

In the additional set of polymorphic SNPs, no association with any variant was found for asthma, allergic rhinitis or eczema. This result is rather consistent with the analysis of further asthma- and atopy-associated traits. Given a sufficient power of this family, samples with highly expressed phenotypes and the absence of any significance, a major effect of STAT3 genomic variants on IgE induction, may be excluded. It makes it even unlikely that such an association exists in the general population as these families represent the upper limit of IgE levels usually prevalent in the general population.

When combining high eosinophil counts and high total IgE levels to a joint HIES-like trait, four SNPs in the $5^{\prime}$-UTR of STAT3 were slightly overtransmitted. This association was restricted to the combination of both traits and did not improve when including a history of atopic dermatitis (data not shown). Given the large number of traits and genotypes tested here, it remains unclear if this association is a finding by chance (in particular, as the associated SNPs are all in linkage disequilibrium and are therefore not independently transmitted) or if it reflects a different STAT3 promoter activity in a minor fraction of asthma children.

The first study of STAT3 variants ${ }^{15}$ unfortunately did not include total IgE as the main outcome. Of the three SNPs associated with FEV1 levels, the first (G3363a16/ rs2306581) was not tested in our study, whereas the second and third associated SNPs (rs957971 and rs1026916) were identical with those associated weakly with the HIES-like trait here. A study of the 1958 British birth cohort reported only online (http://www.b58cgene.sgul.ac.uk/phenoresults. php?gene $=$ stat $3 \&$ pheno $=1$ \&limit $=10$ \&page $=1$ ) did not find any of 19 STAT3 SNPs associated with $\log (\operatorname{IgE})$ values.

Unfortunately, there are also no STAT3 promoter studies available so far that would allow further conclusions. STAT3 RNA levels were uniformly distributed in the published seven HIES patients ${ }^{7}$ and also STAT3 protein levels were similar in EBV-transformed B-cell lines from another six HIES patients. ${ }^{8}$ Nevertheless, as functional STAT3 mutations explained the phenotype of these patients, some of the HIES cases with cryptic origin might have aberrations in the STAT3 promoter as so far only STAT3 cDNA has been sequenced in these patients.

HIES and allergy/asthma are different clinical entities although they may share some clinical symptoms. Some HIES patients will suffer from asthma as well, ${ }^{16}$ whereas asthma patients would score high on the HIES diagnostic scheme ${ }^{17}$ by having raised IgE levels, eosinophil cells and recurrent upper airway infections including pneumonia and eczema. Although minor HIES symptoms like skeletal abnormalities and delay of dentition are not known in atopic patients, ${ }^{18}$ the phenotypic similarities raise the possibility that the heterogenous asthma pool may indeed include some undiagnosed HIES cases.

Airway epithelial STAT3 seems to be required for the allergic inflammation in a murine model of asthma ${ }^{19}$ with the relevance of STAT3 activation during the acute inflammatory response still emerging, ${ }^{20}$ suggesting that this molecule could still be a potential target for regulating the pulmonary inflammatory response.

\section{Acknowledgements}

We thank all participating families for their help and the members of the clinical centers for their work: R Nickel, K Beyer, R Kehrt, U Wahn (Berlin), K Richter, H Janiki, R Joerres, H Magnussen (Grosshansdorf), I M Sandberg, L Lindell, NIM Kjellman (Linkoeping), C Frye, $G$ Woehlke, I Meyer, O Manuwald (Erfurt), A Demirsoy, M Griese, $D$ Reinhardt (München), G Oepen, A Martin, A von Berg, D Berdel (Wesel), $Y$ Guesewell, M Gappa, $H$ von der Hardt (Hannover), $J$ Tuecke, F Riedel (Bochum), M Boehle, G Kusenbach, H Jellouschek, $M$ Barker, G Heimann (Aachen), $S$ van Koningsbruggen, E Rietschel (Köln), P Schoberth (Köln), G Damm, R Szczepanski, T Lob-Corzilius (Osnabrück), L Schmid, W Dorsch (München), M Skiba, C Seidel, M Silbermann (Berlin), A Schuster (Düsseldorf), J Seidenberg (Oldenburg), W Leupold, J Kelber (Dresden), W Wahlen (Homburg), $F$ Friedrichs, K Zima (Aachen), $P$ Wolff (Pfullendorf), D Bulle (Ravensburg), W Rebien, A Keller (Hamburg) and $M$ Tiedgen (Hamburg). We thank M Hoeltzenbein, who helped to start the study, and I Altmüller, who managed the second part of the study, $G$ Schlenvoigt and L Jaeger for IgE determination; L Thaller, G Fischer, $T$ Illig, $N$ Klopp, $C$ Vollmert and $M$ Werner and doctoral students $H$ Gohlke, $N$ Herbon and $G$ Dütsch for their contribution in previous genotyping projects; E André, M Bahnweg, A Luze, C Braig and $B$ Wunderlich for excellent laboratory work. The asthma family study was funded by BMBF 07ALE087, Deutsche Forschungsgemeinschaft DFG WI621/5-1, DFG FR1526/1 and National Genome Network 01GS0122. M Wjst is funded by GSF FE 75051 and the EU Grant Europrevall; BG is supported by the European Commission Marie-Curie Grant MEXT-CT-2006-042316. 


\section{References}

1 Reich NC, Liu L: Tracking STAT nuclear traffic. Nat Rev Immunol 2006; 6: 602-612.

2 Pinto LA, Steudemann L, Depner M et al: STAT1 gene variations, IgE regulation and atopy. Allergy 2007; 62: 1456-1461.

3 Dütsch G, Illig T, Loesgen S et al: STAT6 as an asthma candidate gene: polymorphism-screening, association and haplotype analysis in a Caucasian sib-pair study. Hum Mol Genet 2002; 11: 613-621.

4 Murray PJ: STAT3-mediated anti-inflammatory signalling. Biochem Soc Trans 2006; 34: 1028-1031.

5 Achcar Rde O, Cagle PT, Jagirdar J: Expression of activated and latent signal transducer and activator of transcription 3 in 303 non-small cell lung carcinomas and 44 malignant mesotheliomas: possible role for chemotherapeutic intervention. Arch Pathol Lab Med 2007; 131: 1350-1360.

$6 \mathrm{Fu}$ XY: STAT3 in immune responses and inflammatory bowel diseases. Cell Res 2006; 16: 214-219.

7 Holland SM, DeLeo FR, Elloumi HZ et al: STAT3 mutations in the hyper-IgE syndrome. $N$ Engl J Med 2007; 357: 1608-1619.

8 Minegishi Y, Saito M, Tsuchiya S et al: Dominant-negative mutations in the DNA-binding domain of STAT3 cause hyperIgE syndrome. Nature 2007; 448: 1058-1062.

9 Grimbacher B, Holland SM, Puck JM: Hyper-IgE syndromes. Immunol Rev 2005; 203: 244-250.

10 Altmüller J, Seidel C, Lee YA et al: Phenotypic and genetic heterogeneity in a genome-wide linkage study of asthma families. BMC Pulm Med 2005; 5: 1.

11 Wjst M, Altmüller J, Faus-Kessler T, Braig C, Bahnweg M, Andre E: Asthma families show transmission disequilibrium of gene

variants in the vitamin D metabolism and signalling pathway. Respir Res 2006; 7: 60.

12 Wjst M, Fischer G, Immervoll T et al: A genome-wide search for linkage to asthma. German Asthma Genetics Group. Genomics 1999; 58: 1-8.

13 Ober C, Tsalenko A, Parry R, Cox NJ: A second-generation genomewide screen for asthma-susceptibility alleles in a founder population. Am J Hum Genet 2000; 67: 1154-1162.

14 Dizier $\mathrm{MH}$, Besse-Schmittler C, Guilloud-Bataille $\mathrm{M}$ et al: Genome screen for asthma and related phenotypes in the French EGEA study. Am J Respir Crit Care Med 2000; 162: $1812-1818$

15 Litonjua AA, Tantisira KG, Lake S: Polymorphisms in signal transducer and activator of transcription 3 and lung function in asthma. Respir Res 2005; 6: 52.

16 Renner ED: Klinisch-genetische Definition des Hyper-IgESyndroms (HIES). Thesis Ludwig Maximilians Universität München 2002.

17 Grimbacher B, Holland SM, Gallin JI: Hyper-IgE syndrome with recurrent infections - an autosomal dominant multisystem disorder. N Engl J Med 1999; 340: 692-702.

18 Kankaala TM, Virtanen JI, Larmas MA: Timing of first fillings in the primary dentition and permanent first molars of asthmatic children. Acta Odontol Scand 1998; 56: 20-24.

19 Simeone-Penney MC, Severgnini M, Tu P et al: Airway epithelial STAT3 is required for allergic inflammation in a murine model of asthma. J Immunol 2007; 178: 6191-6199.

20 Gao H, Guo RF, Speyer CL et al: Stat3 activation in acute lung injury. J Immunol 2004; 172: 7703-7712.

Supplementary Information accompanies the paper on European Journal of Human Genetics website (http://www.nature.com/ejhg) 